\title{
Oil Palm Phytochrome-Interacting Factor4 (PIF4) Gene is Conserved and Highly Expressed During Somatic Embryogenesis
}

\author{
Mantira Suksirt ${ }^{1}$, Kamolwan Khianchaikhan ${ }^{1}$, Mya Thuzar ${ }^{2}$, Supachai Vuttipongchaikij ${ }^{1,3}$, Chatchawan Jantasuriyarat ${ }^{1,3^{*}}$ \\ 'Department of Genetics, Faculty of Science, Kasetsart University, Bangkok, Thailand \\ 2Plant Biotechnology Unit, National Center for Genetic Engineering and Biotechnology (BIOTEC-NSTDA), Pathumthani, Thailand \\ ${ }^{3}$ Center for Advanced Studies in Tropical Natural Resources, National Research University-Kasetsart (CASTNAR, NRU-KU), Kasetsart \\ University, Bangkok, Thailand
}

\section{ARTICLE INFO}

Article history:

Received March 20, 2019

Received in revised form September 2, 2019

Accepted September 18, 2019

KEYWORDS:

oil palm,

PHYTOCHROME-INTERACTING FACTOR4,

somatic embryogenesis,

tissue culture

\begin{abstract}
Oil palm is used in food, fuel and cosmetic industries. Tissue culture is the best way to propagate oil palm; unfortunately the somatic embryogenesis during tissue culture takes long time. The molecular mechanism of somatic embryogenesis in oil palm remains unknown. Recent research reported that auxin plays an important role in early and post-embryogenic plant. PHYTOCHROME-INTERACTING FACTOR4 (PIF4) regulates levels of auxin and the expression of key auxin biosynthesis genes. Our research aims to characterize oil palm PIF4 gene. Thus, we cloned $E g P I F 4$, analyzed the domain using bioinformatic and examined the expression of EgPIF4 during somatic embryogenesis at different tissue including callus and somatic embryo stages; globular, torpedo, cotyledon, and plantlet stage using real-time PCR method. The result showed that EgPIF4 gene comprised 1,737 bp with 9 exons, which encode 578 amino acid residuals. It contains a conserved domain called basic helix-loop-helix domain. EgPIF4 has high level of expression at somatic embryogenetic stage specifically globular and torpedo stage suggested that EgPIF4 plays an important role during somatic embryogenesis. The future characterization of $E g P I F 4$ function in oil palm will help to understand somatic embryogenesis process and facilitate the improvement of the oil palm tissue culture.
\end{abstract}

\section{Introduction}

Oil palm (Elaeis guineensis Jacq.) belongs to the Arecaceae family. It is the most important oil crop giving the highest yield per hectare among all oil crops in the world. Palm oil is the source for producing in several industries such as food, fuel and cosmetic. The plantation area in many countries including Indonesia, Malaysia, and Thailand is expanding because of the rapid increase in demand for the oil palm product (Aratrakorn et al. 2006). Tissue culture process are used to propagate high yielding oil palm to meet the demand. Oil palm tissue culture is composed of callus induction, somatic embryogenesis, maturation stage (shoot and root induction) and finally the regeneration of viable plantlets (Thuzar et al. 2011). Normally the production of oil palm by tissue culture technique takes at least one and a half years to obtain small plantlets (Konan et

\footnotetext{
* Corresponding Author

E-mail Address: fscicwj@ku.ac.th
}

al. 2010). The longest stage is somatic embryogenesis stage which takes 7-8 months (Thuzar et al. 2011). Oil palm tissue culture has high economic importance but the biological mechanisms of changes associated with embryogenesis especially at molecular level still unknown.

The most widely used plant growth regulator to control callus induction or somatic embryogenesis induction is 2,4-dichlorophenoxyacetic acid (2,4-D) which is an auxin analog. It is well known that auxin is a key regulator controlling plant cell division and differentiation (Nic-Can and Loyola-Vargas 2016). Auxin plays an important role in early and postembryogenic plant development (Cueva-Agila et al. 2016; Elhiti and Stasolla 2016). From recent researches, the levels of auxin were controlled by PHYTOCHROMEINTERACTING FACTOR4 (PIF4) transcription factor. PIF4 transcription factor regulates levels of auxin and the expression of key auxin biosynthesis gene at high temperature (Franklin et al. 2011). PIF4 and PIF5 
directly controlled auxin biosynthesis and signaling components to ultimately regulate stem growth of plants (Hornitschek et al. 2012). Furthermore, at an early stage of somatic embryogenesis, (Gossypium hirsutum) TEOSINTE BRANCHED1-CYCLOIDEA-PCF transcription factor15, GhTCP15, was phosphorylated. Phosphorylated GhTCP15 has an enhanced binding to promoter of GhPIF4 to regulate the transcription of GhPIF4, so that regulating auxin biosynthesis. During later stage of somatic embryogenesis, GhTCP15 was reduced the phosphorylation activity, thereby decrease auxin biosynthesis, and promote somatic embryo formation (Min et al. 2015).

However, PIF4 gene in oil palm has not been characterized. To understand how PIF4 in oil palm controlling somatic embryogenesis during tissue process, we cloned EgPIF4 gene and examined its expression in different stage of somatic embryogenesis using quantitative real-time PCR. We also investigated the relationship between oil palm and other plant species based on the sequence variation of PIF4 gene. The information from this study will be a preliminary data to show that EgPIF4 may have an important role during somatic embryogenesis stage in oil palm. The investigation and characterization of genes involved in the somatic embryogenesis during tissue culture process will facilitate the understanding of the mechanism and provide the useful information for oil palm improvement and hopefully lead to shortening the length of the oil palm tissue culture process.

\section{Materials and Methods}

\subsection{Plant Materials}

Young leaves of an oil palm Tenera clone from Golden Tenera plantation, Krabi, Thailand were used as an explant for tissue culture based on Thuzar et al. (2011). Oil palm tissues at different developmental stages including callus, somatic embryo at globular, torpedo, cotyledon, and plantlet were used in this study (Figure 1). These samples were collected and kept at $-80^{\circ} \mathrm{C}$ for RNA extraction.

\subsection{RNA Extraction and cDNA Synthesis}

Total RNAs were isolated from oil palm tissue samples including callus, globular, torpedo, cotyledon, and plantlet using Spin Plant RNA (STRATEC Molecular, Germany). Quantity and quality
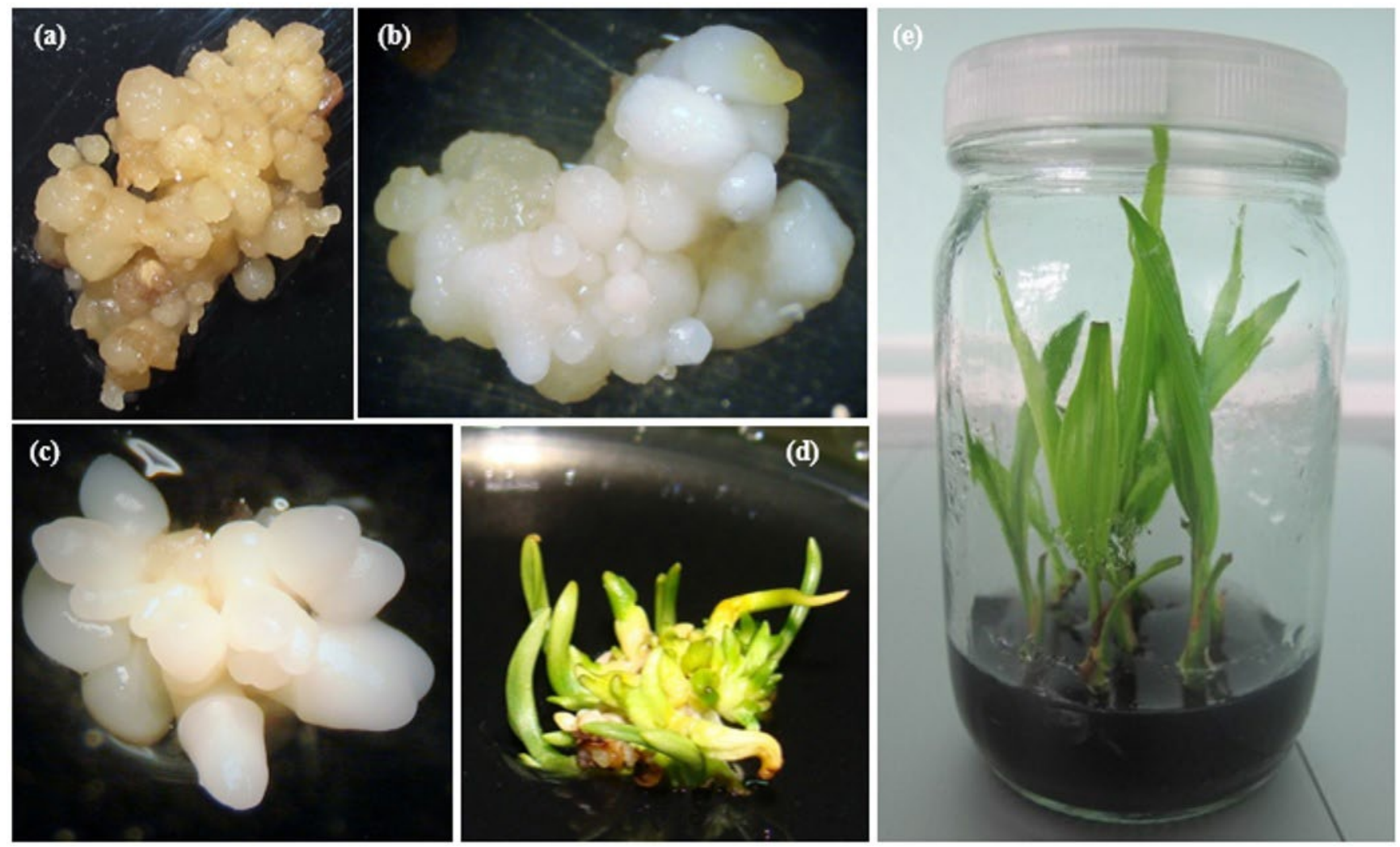

Figure 1. Tissue of oil palm during tissue culture; (a) callus, (b) globular stage, (c) torpedo stage, (d) cotyledonary stage, and (e) plantlet 
of RNAs were assessed using Nanodrop (Thermo Scientific, USA) and agarose gel electrophoresis. The genome DNA was removed by RNAase-free DNAase, then Verso cDNA kit (Thermo Scientific, USA) was used for cDNA synthesis according to the manufacturer's instruction.

\subsection{Cloning of EgPIF4}

Predicted sequence of oil palm PIF4 gene was downloaded from GenBank database(XM_010906351.1). Primer used for gene cloning were EgPIF4_F (5'-ATGAATCACTATGTTCCTCATTG-3') and EgPIF4_R (5'-TTATTGAGTTGAGGGGTACTGG-3'). The PCR was performed as follows: denaturing at $95^{\circ} \mathrm{C}$ for 5 minutes, followed by 35 cycles of amplification $\left(95^{\circ} \mathrm{C}\right.$ for $30 \mathrm{~s}$, $56^{\circ} \mathrm{C}$ for $30 \mathrm{~s}, 72^{\circ} \mathrm{C}$ for $45 \mathrm{~s}$ ) and extension at $72^{\circ} \mathrm{C}$ for 5 minutes (Vivantis, USA). The PCR product fragment was cut out from the gel, extracted and eluted using GF-1 AmbiClean Kit (Gel and PCR) (Vivantis, USA). Finally, the purified DNA was cloned into the pGEM-T easy vector (Promega, USA.). The recombinant vector was transformed to E. coli DH5 $\alpha$ by heat shock method. Recombinant vector from the selected clones were sent out for sequencing at Macrogen, South Korea. The nucleotide sequences obtained from this experiment were annotated by using a BLAST program from the National Center for Biotechnology Information (NCBI) with non-redundant databases (Altschul et al. 1997).

\subsection{Bioinformatics Analysis of EgPIF4 Protein Motif}

The physicochemical properties of the protein encoded by EgPIF4 were predicted with the online Expasy Protparam tool (http://web.expasy.org/ protparam/). The amino acid sequence of PIF4 were downloaded from GenBank database. The multiple sequence alignment of PIF4 amino acid sequences from different plant species were aligned using mafft online sequence alignment (https://mafft.cbrc. jp/alignment/server/). Information of the amino acid sequence of PIF4 gene from 18 plant species used for bioinformatic analysis are shown in Table 1.

\subsection{Analysis of Gene Expression by Quantitative Real-time PCR (qRT-PCR)}

cDNAs from five somatic embryo stages (callus, globular, torpedo, cotyledon, and plantlet) were used for quantitative amplification using EgPIF4 gene specific primers. The primers used for EgPIF4 gene expression were PIF4_F (5'-AACACAATGCCACCTCCTAA-3') and PIF4_R (5'-GCCACCTGATGATGAAGTAAC-3'). Realtime qPCR was performed on a LightCycler 480
Table 1. Information of the amino acid sequence of PIF4 gene from 18 plant species used for bioinformatic analysis

\begin{tabular}{|c|c|c|}
\hline Common name & Sciencename & Accession no. \\
\hline Arabidopsis & Arabidopsis thaliana & NP_001323428.1 \\
\hline green algae & $\begin{array}{l}\text { Coccomyxa } \\
\text { subellipsoidea }\end{array}$ & XP_005644417 \\
\hline cucumber & Cucumis sativus & XP_011653988 \\
\hline oil palm & Elaeis guineensis & XM_010906351.1 \\
\hline rose gum & Eucalyptus grandis & KCW89798 \\
\hline blue lupin & $\begin{array}{l}\text { Lupinus } \\
\quad \text { angustifolius }\end{array}$ & XP_009757613.1 \\
\hline tobacco & Nicotiana sylvestris & XP_009757613.1 \\
\hline rice & Oryza sativa & XP_015631787.1 \\
\hline date palm & Phoenix dactylifera & XP_008811817.2 \\
\hline desert poplar & Populus euphratica & XP_011026200.1 \\
\hline peach & Prunus persica & XP_020416275.1 \\
\hline sesame & Sesamum indicum & XP_011100372.1 \\
\hline tomato & Solanum pennellii & XP_027774395.1 \\
\hline sorghum & Sorghum bicolor & XP_021302236.1 \\
\hline cacao & Theobroma cacao & XP_017971849.1 \\
\hline mungbean & Vigna radiata & XP_014522272.1 \\
\hline grape & Vitis vinifera & XP_010657098.1 \\
\hline corn & Zea mays & NP_001348421.1 \\
\hline
\end{tabular}

(Roche, Switzerland) in 96 well plates in $10 \mu$ reaction volume containing $2 \mu$ of 1/40 time dilution cDNA, $1.5 \mu \mathrm{l}$ of $2 \mu \mathrm{M}$ for each primer and $5 \mu \mathrm{SYBR}^{\circledR}$ Green Master mix (Roche, Switzerland). Real-time PCR was initiated by denaturation at $95^{\circ} \mathrm{C}$ for 10 minutes, followed by 45 cycles of $95^{\circ} \mathrm{C}$ for $15 \mathrm{~s}, 60^{\circ} \mathrm{C}$ for $15 \mathrm{~s}$, and $70^{\circ} \mathrm{C}$ for 1 minute. Expression was normalized with the amplification of oil palm elongation factor gene; $E g E F 1-\alpha \_F(5$ '-ACATTGTCGTCATTGGTCAT-3') and EgEF1- $\alpha \_R(5$ '-GGGTAAAGGCAAGCAAAGCA-3')(EgEF1- $\alpha$, accession number: NM_001303577). Amplification of RNA matrices were also conducted to validate the absence of DNA in each sample. The gene expression experiment was performed in triplicate using two independent biological samples.

\section{Results}

\subsection{Conservation of a Basic Helix-loop-helix Domain in Plant Species}

Somatic embryo tissue of oil palm was used for RNA extraction including callus, globular, torpedo, cotyledon, and plantlet stage (Figure 1). EgPIF4 gene was cloned using cDNA from globular tissue during somatic embryogenesis process. The nucleotide sequence was blast against the oil palm genome database. The results showed that EgPIF4 gene located on chromosome 1 in oil palm genome. The coding sequence of EgPIF4 gene comprised 1,737 bp with 9 exons (Figure 2a). EgPIF4 protein comprises of 578 amino acid residuals, 
which contains a basic helix-loop-helix domain (bHLH domain) in the amino acid position 346-413 (Figure 2b). Analysis of physicochemical properties revealed a protein molecular weight of $63.71 \mathrm{kDa}$ and an isoelectric point of 6.48 .

Since the amino acid sequence of PIF4 proteins from different plant species has no conserved domain at the start and the middle of the sequence, an alignment of a conserved amino acid sequence of EgPIF4 protein and PIF4 proteins from different plant species is shown in Figure 3 . The amino acid sequence alignment revealed that PIF4 protein contains a conserved structural domain, namely bHLH domain. The result showed that the deduced amino acid sequence of EgPIF4 was highly similar to that of other PIF4 proteins.

\subsection{EgPIF4 Expressed During Somatic Embryogenesis in Oil Palm Tissue Culture}

To investigate the EgPIF4 expression levels at different developmental stages during oil palm tissue culture, a qRT-PCR experiment was performed. From the result, transcript level of EgPIF4 when

\section{5 'UTR} ATG

b

CDS atgaatcactatgttcctcattggaacttggaagatgagacaacatcttcggaggacttccagcgacaaatcagaaaaaactatctgg

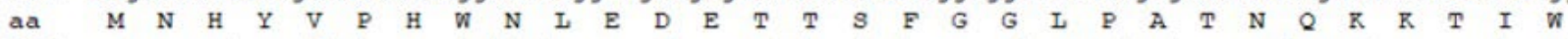
CDS ccggacaatgagcttgtagagctcttgtggcaagatgggegtgtaatcatgcacagtcagactcatcggaaatcccctcccaccgtcggt

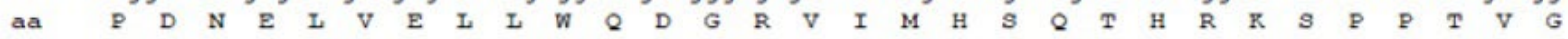
CDS gaatttaaacaagctcagaaggctgagccagcatcaaaatgcaggggggctctcgggaattcgagcaatttgagtcaagaagatgaaact

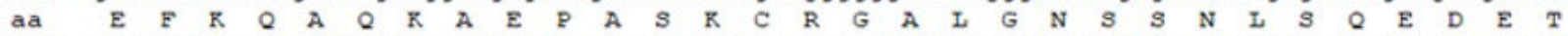
CDS gcctcatggttccaacactctctcgatgattcgctagaaaagagttctcagaattctttgcgaaagccaalgtagatgceattggt

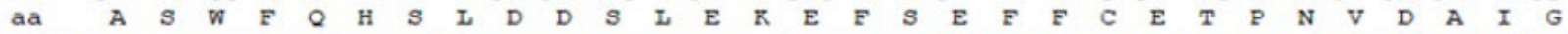
CDS agcagcaagatgactaaagatgtcactgaagagggagagagatccacaaacttggtgegaatgaggaaacgaatgtgtttgctgcttct

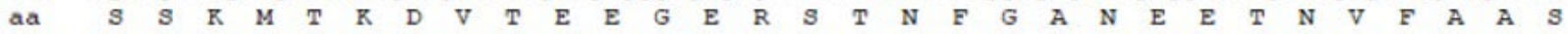
CDS gatcccaagcaatccaggatttgtttcaaggaaaacacaatgccacctcctaaatcccacattattgcttctacccagcaggcttcttgt

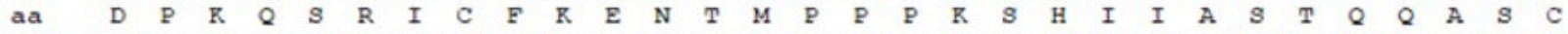
CDS ttggcaaatagcaatctcgtaaattttcacacttcacaaggccacggccggtgatggctgatgtgggatcattaaatgggcagctgggg

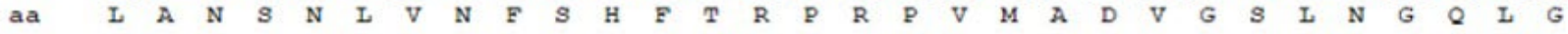
CDS aaggaatcagggaagagaattcgggcaggggcggtagagtccacctcgacgatgaccattgcatcaagtatctgtgagagcaaccagatt

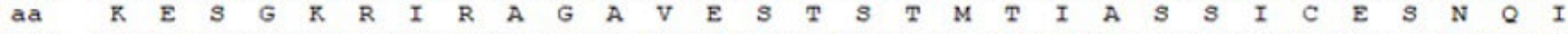
CDS caagctcaggccgatccaagccatactataagcagtgatgctgcaggagttgttgtaagaggtctgaaggaggacgeccagatgacatca

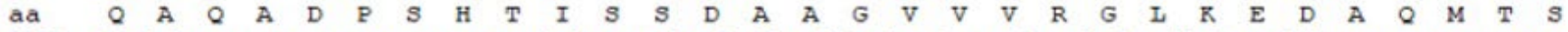
CDS ctatctgagagaacacgaacgaacacgtatgaagctactgttacttcatcatcaggtggctctggttgtagcttggaagaacaggacag

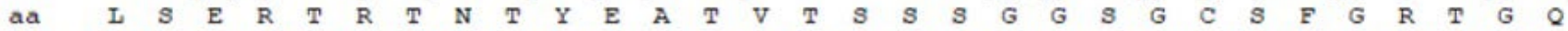
CDS cagagtgecatcaatcagagccacaaaagaaagggaagagatgcagaggaatatgaaagccagagcgaggaggctgactatgagtatgtc

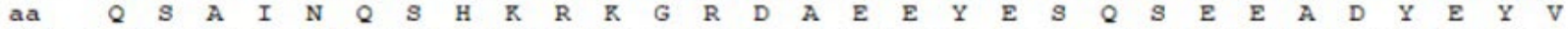
CDS gaggcaaacaagctagcgcagcggtctgcattgccacgaagaagccgttctgctgaagtccacaatctctccgagaggagaagaagggac

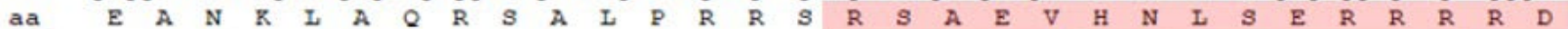
CDS agaataatgaaagatgaaggcactacaagagctcatacctcattgcaacaagctactttattctcggaacatcggacatcgtcatgat

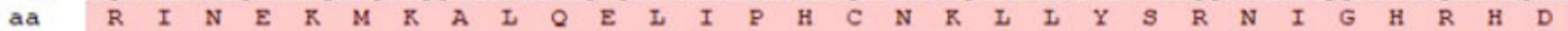
CDS gtaatcaagtttgtaataacagacaagcatcgatgctagatgaggcaattgagtacttgaagtccettcaactgcaagttcagataatg

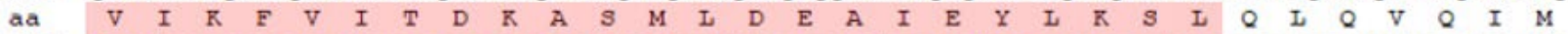
CDS tggatgggaagtggcatggcaccaatgatgtttcetgctgtccaacaatgcatgtcacgcatcggtatgggagtgggtcatgcttctatg

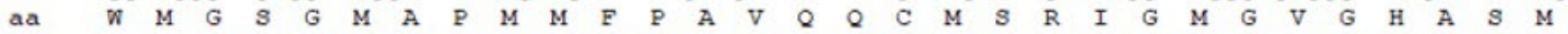
CDS ccetctatgcatggtccagtccaattgecaagagtcceatttctcaatcagtctatagectcggettcgacggeacaccagacacegete

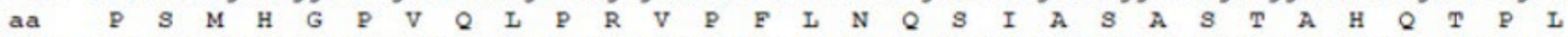
CDS tgcctatcaacggccttgaatgcattgaacttccaaatcagatgcagaatatccatcttcctggttcgtatgccecataccttggcttc

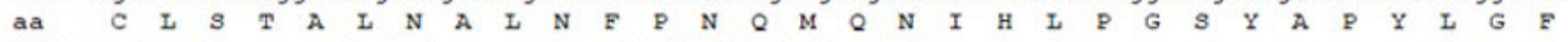
CDS catcacatgcaagcatcctctcaggaatgaattgttgtacctatggatctcacatggtgcagcagaatccgacaacagcagtgccgagc

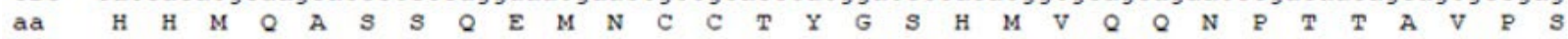
CDS agcagcatcttcctactgctggagaagttccegctgagaataatcaaataacaaatctggatctgcaaatggttcacattagtggg

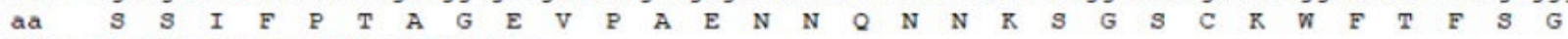
CDS tccetccagtaccctcaactcaataa

aa $S$ L $Q$ Y $P$ S T Q

Figure 2. Characteristic of EgPIF4 gene. (a) Gene structure of EgPIF4 gene contain 9 exons of 1,737 bp and (b) coding sequence and amino acid sequence of EgPIF4 gene. Red color highlight indicates the bHLH domain. Dash (-) refers to stop codon 
compared with the expression level of EgEF1- $\alpha$, was not detected in in callus but detected in all somatic embryo tissue (globular, torpedo, and cotyledon), with the highest relative expression observed in globular stage. The relative expression of EgPIF4 was then gradually decreased after globular stage (Figure 4). This result suggested that EgPIF4 may play an important role at somatic embryogenesis process during tissue culture.

\section{Discussion}

Since clonal propagation is the best way to propagate high yield oil palm, tissue culture technique was used.

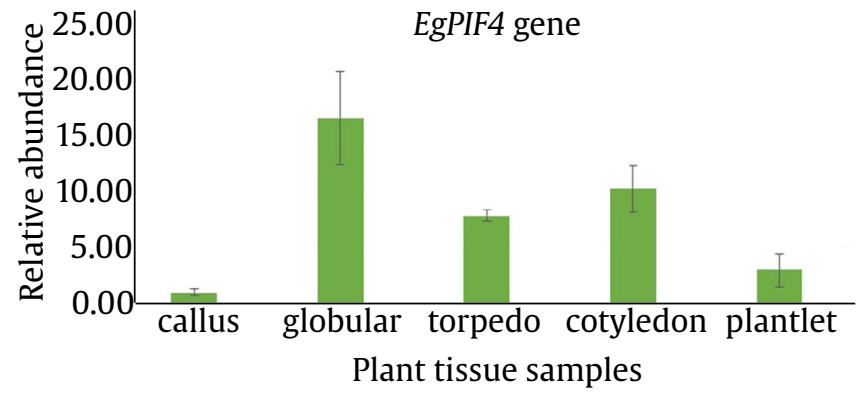

Figure 4. Quantitative real-time PCR analysis of EgPIF4 transcription levels in oil palm tissue samples including somatic embryo tissues: callus, globular, torpedo, cotyledon, and plantlet. Oil palm elongation factor gene, $E g E F 1-\alpha$, was used as internal control

bHLH domain

\begin{tabular}{|c|c|c|}
\hline Arabidopsis & GNKSNQRSGSNRRSRAAEVHILSE--RRRRDRI-- & 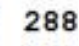 \\
\hline Jucumis & NKTAPRSGSSRRTRAAEVHNLSE--RRRRERI-C & 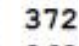 \\
\hline Prunus & GNKSAQRSGSSRRSRAAEVHNLSE--RRRRDRI- & \\
\hline Vitis & RNKASQRSGSTRRSRAAEVHNLSE--RRRRDRI----- & 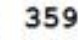 \\
\hline opulus & ANKPAKRSGSARRSRAAEVHNLSE--RRRRDRI-------- & 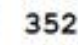 \\
\hline Theobroma & AGNKPTQRSGSSRRSRAAEVHNLSE--RRRRDRI---1--- & 36 \\
\hline Eucalyptus & GGKKPATRSGSTRRSRAAEVHNLSE--RRRRDRI-------- & 39 \\
\hline tiana & GNKSAQKSGTARRSRAAEVHNLSE--RRRRDRI------ & 32 \\
\hline Solanum & GGNKPAQKSGTARRSRAAEVHNLSE--RRRRDRI & 30 \\
\hline Lupinus & IGSKASQHAGSSRRNRAAEVHNLSE--RRRRDRI- & 36 \\
\hline ig & GNKTSQRTGSARRNRAAEVHNLSE- RRRRDRI- & 37 \\
\hline Sesamum & GNKSSKKSGTTRRNRVAEMHNLSE--RRRRDRI-- & 33 \\
\hline Ela & ANKLAQRSALPRRSRSAEVHNLSE--RRRRDRI--------------NEKIKIKALQE & 38 \\
\hline$i x$ & EANKLAQRSPFTRRSRSAEVHNLSE--RRRRDRI---- & 43 \\
\hline Sorghum & TTCEPAHKTATAKRRRAAEVHNLSE--RRRRDRI--------------NEKMKALQE & 35 \\
\hline zea & PPVPARPLPGPARRLFAALRLRLRLRSMTRRNTFTAANAGRGAGVRAGAEDDDCQAAPRR & 28 \\
\hline Coccomyxa & 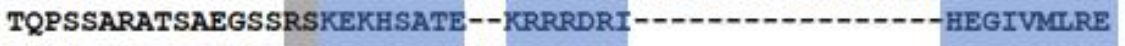 & 78 \\
\hline \multirow[t]{2}{*}{ Oryza } & 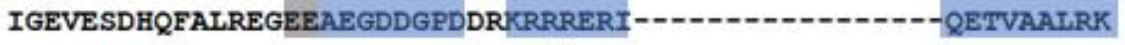 & \\
\hline & \multicolumn{2}{|l|}{ bHLH domain $^{* *}$ : : } \\
\hline is & -IIPHCSK------------------TDKASILDEAIDYLKSLQ LQLQVMW-MGSGMAAA & 329 \\
\hline mis & -LIPHCNK------------TDKASMLDEAIEYLKSLQLQLQVMW-MGSGM--- & 413 \\
\hline Prunus & -LIPHSNK------------TDKASMLDEAIEYLKSLQMQLQVMW-MGSGM--- & 409 \\
\hline Vitis & -LIPHSNK-----------SDKASMLDEAIEYLKSLQLQLQLMW-MGGGV--- & 400 \\
\hline Populus & - & 393 \\
\hline Theobroma & -LIPHCNK-----10--- & 401 \\
\hline Eucalyptus & -LIPHCNK----- TDKASMLDEAIEYLKSLQLQLQVMW-MGGGM--- & 434 \\
\hline Nicotiana & -LLPHSTK-------- TDKASMLDEAIEYLKSLQMQLQMMN-MGSGM--- & 361 \\
\hline Solanum & -LLPHSTK----------1DKASMLDEAIEYLKSLQMQLQMMW-MGSGM--- & 393 \\
\hline Lupinus & -LIPNSNK---------- & 408 \\
\hline Vigna & -LIPHSSK--------------TDKASMLEEAIEYLKSLQLQLQLMW-MGSGM--- & 412 \\
\hline Sesamum & -LIPHSNK--------------SDKASMLDEAIEYMKSLQMQLQWMW-MGSGM--- & 371 \\
\hline Ela & -LIPHCNKLLYSRNIGHRHDVIKFVITDKASMLDEAIEYLKSLQLQVQIMW-MGSGM--- & 426 \\
\hline Phoenix & -LIPHCNK-----------TDKASMLDEAIEYLKSLQLQVQIMW-MGNGM--- & 473 \\
\hline sorghum & -LIPHCNK------1 & 392 \\
\hline Zea & 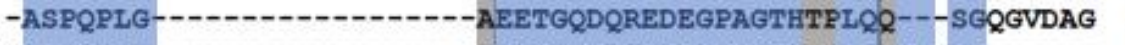 & 341 \\
\hline Coccom & VVVPQKEK------1--- & 119 \\
\hline Oryza & 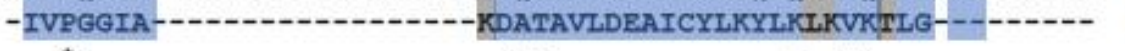 & 311 \\
\hline
\end{tabular}

Figure 3. Sequences alignment between the deduced amino acid of EgPIF4 and other homologous genes in plants. Blue background represents amino acid identity is over $80 \%$, and gray background represents amino acid identity is over $50 \%$, The bHLH domains is marked with an overline 
Unfortunately, the somatic embryogenesis during tissue culture process in oil palm take longer time that other plants. Genes involved in this process were studied. PIF4 is a transcription factor that was reported to have a role in controlling the level of auxin which is an important hormone during somatic embryogenesis process. The molecular mechanisms of PIFs have been studied extensively in Arabidopsis. Auxin is an important plant hormone essential for many aspects of plant growth and development and also has an essential role during somatic embryogenesis. It plays an important role in early and post-embryogenic plant development (Cueva-Agila et al. 2016; Elhiti and Stasolla 2016). The basic helix-loop-helix (bHLH) transcription factor phytochrome-interacting factor 4 (PIF4) has a key role in controlling the levels of auxin. PIF4 was reported to be a regulator that control levels of auxin and the expression of key auxin biosynthesis gene at high temperature (Franklin et al. 2011). However, not many studies focus on the role of PIF4 during somatic embryogenesis and PIF4 gene in oil palm has not been characterized. The results from our studies showed that EgPIF4 is located on chromosome 1 and contains 1,737 bp open reading frame encoding a polypeptide comprised of 578 amino acid residuals.

The results from a database search revealed that PIF4 contains a region with strong homology to the bHLH superfamily of transcription factors (Evan and Littlewood 1998; Atchley et al. 1999). From our results, sequence comparison of the bHLH domain from different plant species revealed that the residues that define the bHLH domain are conserved in the PIF4 amino acid sequence. This suggested that EgPIF4 transcription factor may play an important role during embryo development as in other plants.

Recent researches reported the expression of PIF4 gene in various tissue. PIF4 was found to be significantly induced at the early stage of leaf senescence and decreased at the late stage of leaf senescence in Arabidopsis (Song et al. 2014). RT-qPCR analysis showed that PIF4 gene in maize is expressed in roots, stems, coleoptiles, and leaves at the six-leaf stage (Shi et al. 2018). PIF4 gene in oil palm has not been studied and characterized before. From our studies, we showed that EgPIF4 gene in oil palm, specifically expressed in somatic embryo tissues with the highest expression at globular and cotyledon stages which are the somatic embryogenesis stage. Furthermore, EgPIF4 showed no expression at callus stage. These results supported the idea that PIF4 gene is the important regulator for the embryogenesis in plant species including oil palm.
The results from this study were a preliminary data to show that EgPIF4 may plays an important role during somatic embryogenesis. EgPIF4 protein will be used for characterizing the function and the role during somatic embryogenesis in the future.

\section{Acknowledgements}

This work is supported by grants from The Thailand Research Fund (TRF), The Commission on Higher Education and Kasetsart University Research and Development Institute(KURDI)(No. RSA5980046). Miss Mantira Suksirt received the National Research Council of Thailand (NRCT) in the 2018 fiscal year and a Science Achievement Scholarship of Thailand and financial support to conduct part of this study from The Capacity Building of KU Students on Internationalization Program: KUCSI, the International Affairs Division, Kasetsart University.

\section{Conflict of Interest}

The authors report no conflict of interest.

\section{References}

Altschul SF et al. 1997. Gapped BLAST and PSI-BLAST: a new generation of protein database search programs. Nucleic Acids Research 25:3389-3402.

Aratrakorn S et al. 2006. Changes in bird communities following conversion of lowland forest to oil palm and rubber plantations in southern Thailand. Bird Conservation International 16:71-82.

Atchley WR et al. 1999. Positional dependence, cliques, and predictive motifs in the bHLH protein domain. Journal of molecular evolution 48:501-516.

Cueva-Agila AY et al. 2016. Effects of plant growth regulator, auxin polar transport inhibitors on somatic embryogenesis and CMSERK gene expression in Cattleya maxima (Lindl.). In: Mujib A. (Eds.). Somatic Embryogenesis in Ornamentals and Its Applications. New Delhi: Springer. pp. 255-267.

Elhiti M, Stasolla C. 2016. Somatic embryogenesis: the molecular network regulating embryo formation. In: Mujib A. (Eds.). Somatic Embryogenesis in Ornamentals and Its Applications. New Delhi: Springer. pp. 217-229.

Evan G, Littlewood T. 1998. A matter of life and cell death. Science 281:1317-1322.

Franklin KA et al. 2011. Phytochrome-interacting factor 4(PIF4) regulates auxin biosynthesis at high temperature. Proc Natl Acad Sci USA 108:20231-20235.

Hornitschek P et al. 2012. Phytochrome interacting factors 4 and 5 control seedling growth in changing light conditions by directly controlling auxin signaling. The Plant Journal 71:699-711. 
Konan KE et al. 2010. In vitro conservation of oil palm somatic embryos for 20 years on a hormone-free culture medium: characteristics of the embryogenic cultures, derived plantlets and adult palms. Plant cell 29:1-13.

Min L et al. 2015. Leafy cotyledon1-casein kinase I-TCP15phytochrome interacting factor4 network regulates somatic embryogenesis by regulating auxin homeostasis. Plant physiology 169:2805-2821.

Nic-Can GI, Loyola-Vargas VM. 2016. The role of the auxins during somatic embryogenesis. In: Loyola-Vargas V, Ochoa-Alejo N (Eds.). Somatic embryogenesis: fundamental aspects and applications. Cham: Springer. pp. 171-182.
Shi Q et al. 2018. Functional characterization of the maize phytochrome-interacting factors PIF4 and PIF5. Frontiers in Plant Science 8:2273. DOI:10.3389/fpls.2017.02273

Song $\mathrm{Y}$ et al. 2014. Age-triggered and dark-induced leaf senescence require the bHLH transcription factors PIF3, 4, and 5. Molecular plant 7:1776-1787.

Thuzar M et al. 2011. Efficient and rapid plant regeneration of oil palm zygotic embryos cv. 'Tenera' through somatic embryogenesis. Acta Physiologiae Plantarum 33:123-128. 